\title{
Pedicularis resupinata restores corticosterone- induced depressive-like behaviors in mice via glucocorticoid receptor-mediated BDNF expression
}

\author{
Dong Wook Lim \\ Korea Food Research Institute
}

Daeseok Han

Korea Food Research Institute

Changho Lee ( $\nabla$ chang@kfri.re.kr)

Korea Food Research Institute https://orcid.org/0000-0002-1039-1434

\section{Research Article}

Keywords: Pedicularis resupinata, acteoside, depression, glucocorticoid recepto, BDNF

Posted Date: February 22nd, 2022

DOI: https://doi.org/10.21203/rs.3.rs-1348193/v1

License: (c) This work is licensed under a Creative Commons Attribution 4.0 International License.

Read Full License 


\section{Abstract}

Excessive corticosterone (CORT), resulting from a dysregulated hypothalamic-pituitary-adrenal (HPA) axis, is associated with cognitive impairment and behavioral changes, including depression. In Eastern medicine, Pedicularis resupinata var. oppositifolia (Scrophulariaceae) is used for the treatment of rheumatoid arthritis, malignant abscesses (tumors), uroliths, and diuretics. However, the antidepressant properties of $P$. resupinata have not been well characterized. Here, the antidepressant-like effects of $P$. resupinata extract (PRE) were evaluated in in vivo and in vitro CORT-induced depression models. HPLC confirmed that acteoside, a phenylethanoid glycoside, was a major PRE compound from. Male ICR mice (8 weeks old) were injected with CORT ( $40 \mathrm{mg} / \mathrm{kg}$ ) and orally administered PRE daily $(30,100$, and 300 $\mathrm{mg} / \mathrm{kg}$ ) for 21 consecutive days. Depressive-like behaviors were evaluated using the open field test, sucrose preference test, passive avoidance test, tail suspension test, and forced swim test. Treatment with a high dose of PRE significantly alleviated CORT-induced depression-like behaviors in mice and reduced neurotoxicity in a concentration-dependent manner in SH-SY5Y cells. Additionally, repeated CORT injection markedly reduced brain-derived neurotrophic factor (BDNF) levels, whereas total glucocorticoid receptor (GR) and GR phosphorylation at serine 211 were significantly increased in the mice hippocampus, but improved by PRE treatment. Thus, our findings suggest that PRE have potential antidepressant-like effects by modulating GR-mediated BDNF expression in the CORT-induced depressive mice model.

\section{Introduction}

Depression is one of the most serious global health concerns, with over 300 million individuals affected worldwide (Ali et al., 2021). However, currently available first-line antidepressants are not completely effective and can cause a variety of undesirable side effects (Racagni and Popoli, 2010). Therefore, alternative approaches, including herbs such as Hypericum perforatum (i.e., St. John's wort) (Pirotta et al., 2014) or increased intake of antioxidants via fruits and vegetables, have attracted interest as potential antidepressants (X. Q. Liu et al., 2016). Pedicularis resupinata, an edible Korean wild plant belonging to the family Scrophulariaceae, has been used as an anti-inflammatory compound in traditional Korean medicine (Y. M. Lee et al., 2011). Although little is known about the pharmacological efficacy of $P$. resupinata, the extract of the species of Pedicularis, which contains phenylpropanoid glycoside and acteoside as the main components, is known for its antioxidant effects (P. Wang et al., 1996). Several studies have reported that acteoside had antioxidant (H. D. Lee et al., 2020), anti-inflammatory (Qiao et al., 2019), neuroprotective (H. Q. Wang et al., 2009), and hepatoprotective (Cui et al., 2018) properties. However, the antidepressant-like effects of $P$. resupinata extract on depressive mood have not yet been defined. In order to explore natural extracts with antidepressant efficacy, we conducted an in vitro assay (Lim et al., 2018a) to evaluate the neuroprotective effects of several Korean wild plants, among which $P$. resupinata extract showed a high neuroprotective potential, and it was determined that acteoside was the main component of $P$. resupinata extract. In this study, we aimed to investigate the antidepressant-like 
effects of $P$. resupinata extract (PRE) in an animal model of depression established by repeated chronic administration of corticosterone (CORT).

\section{Material And Methods}

\section{Sample preparation}

$\mathrm{MeOH}$ extract of Pedicularis resupinata (PRE, voucher specimen No. KPM032-051) was purchased from the Korea Plant Extract Bank of the Korea Research Institute of Bioscience and Biotechnology (Daejeon, South Korea). PRE was dissolved in 50\% HPLC-grade MeOH and analyzed by HPLC (Jasco, Tokyo, Japan) using a Waters Symmetry ${ }^{\circledR} \mathrm{C} 18$ column $(4.6 \times 250 \mathrm{~mm}, 5 \mu \mathrm{m})$. The mobile phase was established using $0.2 \%$ formic acid $(A)$ and $A C N / M e O H(60: 40, v / v)(B)$, by gradient elution. The chromatographic separation was processed with a solvent (A) gradient that decreased from $80 \%$ to $50 \%$ within 40 min of running time, and then increased to $80 \%$ with a 45 min running time. The injection volume of PRE was $10 \mu \mathrm{L}$ with a flow rate of $1 \mathrm{~mL} / \mathrm{min}$, and detected at $280 \mathrm{~nm}$ using a PDA detector. The concentration of acteoside was $0.78 \pm 1.15$ (mean \pm SD) $\mathrm{mg} / \mathrm{g}$. Representative chromatograms of acteoside and PRE are shown in Fig. 1

\section{Animal and treatments}

ICR mice (male, 7 weeks old, weighing 25-28 g) were purchased from KOATECH Animal Inc. (Pyeongteak, South Korea). The mice had ad libitum access to food and water in a climate-controlled chamber (temperature, $21 \pm 2{ }^{\circ} \mathrm{C}$ and light-dark cycle, $24 \mathrm{~h}$, lights on at 07:00, and lights off at 19:00). All mice ( $\mathrm{n}=$ 4 per cage) were habituated to the facility for 1 week prior to starting the experiments. All animal experiments were approved by the Institutional Animal Care and Use Committee of the Korea Food Research Institute (KFRI-M-19016). To induce depression-like behavior, a high-dose of CORT was intraperitoneally (i.p.) injected according to a previous study. CORT (Sigma-Aldrich, MO, USA) was dissolved in $0.9 \%(\mathrm{w} / \mathrm{v})$ saline containing $0.1 \%$ dimethyl sulfoxide (DMSO; Sigma-Aldrich) and $1 \%$ Tween80. St. John's wort ( $80 \% \mathrm{MeOH}$-extract dried powder of Hypericum japonicum), which was the positive control, and PRE was dissolved in distilled water. The mice were randomly assigned to six groups $(n=8)$ : (1) sham, (2) control, (3) St. John's wort $300 \mathrm{mg} / \mathrm{kg}$, (4) PRE $30 \mathrm{mg} / \mathrm{kg}$, (5) PRE $100 \mathrm{mg} / \mathrm{kg}$, and (6) PRE $300 \mathrm{mg} / \mathrm{kg}$. Mice in the control and sample-treated groups were orally administered CORT $(40 \mathrm{mg} / \mathrm{kg}$, i.p.) once daily, while those in the sham group were treated with an equal volume of vehicle. After 3 weeks of treatment, the mice tested in depression-related behavioral tasks, beginning $1 \mathrm{~h}$ after sample administration, as per the experimental scheme (Fig. 2), and were then sacrificed for western blot analysis.

\section{Open filed test (OFT)}

The mice were placed in the center of an open field maze $(50 \times 50 \times 50 \mathrm{~cm})$, and their movements were recorded using a video camera; behaviors, total distance, and time in the center and periphery zones were tracked for 5 min using the SMART video tracking sys-tem (SMART v3.0, Panlab SL, Barcelona, Spain). 


\section{Sucrose preference test (SPT)}

The SPT was performed 24 hours after OFT, following a previously established protocol (Lim et al., 2019a). The two bottles contained the $1 \%$ sucrose solutions were located in the cage for $24 \mathrm{~h}$. Next, one of the bottle of the sucrose solution was located with water for $24 \mathrm{~h}$. The mice then were placed in cages individually with free access to the two bottles for $24 \mathrm{~h}$, and the consumed volumes were recorded. Sucrose preference was calculated as follows: consumption $(\%)=[$ sucrose intake/(sucrose intake + water intake)] $\times 100$.

\section{Passive avoidance test (PAT)}

The PAT was performed using the passive avoidance apparatus (GEMINI, SD instruments, San Diego, CA, USA), as previously described. The PAT experiment was conducted across 2 days. In the training trial, each mouse was placed in a safe zone with a closed door. After acclimatization for $1 \mathrm{~min}$, the door opened automatically, and the mice were allowed to enter the dark zone. When the mice entered the dark zone, an electrical foot shock of $0.5 \mathrm{~mA}$ for $3 \mathrm{~s}$, was elicited. On the next day, the mice were again placed in the safe zone, and the latency to enter the dark zone was recorded.

\section{Tail suspension test (TST) and forced swim test (FST)}

In the case of the TST, each mouse was suspended by its tail using adhesive tape and attached to a hook that automatically measured their movement. The immobility time during the 6-min test was measured using an automated TST apparatus (BioSeb, Chaville, France). In the FST, the mice were forced to swim for $6 \mathrm{~min}$ in a transparent Plexiglas cylinder (height, $13 \mathrm{~cm}$; diameter, $24 \mathrm{~cm}$ ) filled with water to a depth of $10 \mathrm{~cm}$ (temperature, $22-24^{\circ} \mathrm{C}$ ). The immobility time during the last 4 min was measured using a SMART video tracking system (SMART v3.0, Panlab SL, Barcelona, Spain).

\section{Cell viability assay}

Cell viability was analyzed using the 3-(4, 5-dimethylthiazol-2-yl)-2, 5-diphenyltetrazolium bromide MTT assay, as previously described. SH-SY5Y cells (ATCC, Manassas, VA, USA) were cultured in Dulbecco's modified Eagle's medium (Gibco, NY, USA) supplemented with 10\% fetal bovine serum (Gibco) and 1\% penicillin-streptomycin-glutamine (Gibco). The cells were maintained in a humidified atmosphere containing $5 \% \mathrm{CO}^{2}$ at $37^{\circ} \mathrm{C}$. The cells were seeded in 96 -well plates $\left(2 \times 10^{4}\right.$ cells/well $)$ and incubated for $24 \mathrm{~h}$. To assess the effect of PRE on cell viability, a MTT assay was used to determine the viability of cells treated with CORT. Cells were seeded at a density of $2 \times 10^{4}$ cells/well in 96-well plates and incubated overnight. The next day, the cells were co-treated with 10,50 , or $100 \mu \mathrm{g} / \mathrm{mL}$ of PRE and CORT $(200 \mu \mathrm{m})$ for $24 \mathrm{~h}$. MTT solution (thiazolyl blue tetrazolium bromide, M5655, Sigma-Aldrich, MO, USA) was added to each well at a final concentration of $0.5 \mathrm{mg} / \mathrm{mL}$, and the plates were incubated at $37^{\circ} \mathrm{C}$ for $4 \mathrm{~h}$. The medium was then discarded, and the formazan product was dissolved in DMSO (Sigma-Aldrich). Cell viability was determined by measuring the optical density at $570 \mathrm{~nm}$ wavelength. 


\section{Western blotting}

Hippocampal brain tissues were homogenized in RIPA buffer containing a protease and phosphatase inhibitor cocktail. The quantified proteins $(20 \mu \mathrm{g})$ were separated by $10 \%$ sodium dodecyl sulfatepolyacrylamide gel electrophoresis and transferred onto polyvinylidene fluoride membranes (Millipore, Billerica, MA, USA). The membranes were blocked with $4 \%$ skim milk in Trisbuffered saline with $1 \%$ Tween-20 for 40 min, and subsequently probed with the following primary antibodies: BDNF mouse monoclonal antibody (1:1,000 dilution, sc-65514, Santa Cruz Biotechnology, CA, USA), glucocorticoid receptor (1:1,000 dilution, sc-393232, Santa Cruz Biotechnology, CA, USA; GR phosphorylation at serine 211 (Ser211, 1:1,000 dilution, \#4161, Cell Signaling, MA, USA), and an-ti- $\beta$-actin rabbit polyclonal antibody (1:1,000 dilution, \#4967, Cell Signaling, MA, USA) overnight at $4{ }^{\circ} \mathrm{C}$ in $3 \%$ skim milk in TBST. After incubation with the horseradish peroxidase linked secondary antibody for $2 \mathrm{~h}$, immunoreactive proteins were detected using a chemiluminescence detection system (LI-COR Biosciences, Lincoln, NE, USA) and then analyzed using ImageJ software (NIH, Bethesda, MD, USA).

\section{Statistical analyses}

Statistical analyses were performed using Student's t-test for two-sample comparisons and one-way analysis of variance, followed by Tukey's post-hoc test using Prism 8 (GraphPad Software, Inc., San Diego, CA, USA) for multigroup comparisons. All data are presented as the mean \pm SEM. Differences were considered significant at $p<0.05$.

\section{Results}

\section{Effect of PRE on OFT in CORT-injected mice}

First, we examined the effect of PRE on CORT-induced alterations in the open field test (OFT). As shown in Figure 3, CORT-injected mice did not show a significant change in locomotor activity (Fig. 3 A). In addition, there was no significant difference in the total distance moved (Fig. 3B), time spent in the center (Fig. 3C), or periphery (Fig. 3D) of the OFT, between the PRE-treated groups.

\section{Effect of PRE on SPT}

The repeated CORT injection significantly decreased the percentage of sucrose consumption as compared with the sham group $(p<0.05)$. However, it was showed that the reduced percentage of sucrose consumption was significantly restored in the St. John's wort treated group $(p<0.05)$. Similarly, PRE at a dose of $300 \mathrm{mg} / \mathrm{kg}$ significantly increased the percentage of sucrose consumption $(P<0.05)$ (Fig. 4).

\section{Effect of PRE on PAT}

Memory impairment are commonly seen in many stress-related disorders, including depression (Kalueff and Murphy, 2007). Thus, we examined the effect of PRE on memory impairment caused by CORT 
injection in mice. As shown in Figure 5, CORT-injected mice exhibited memory impairment as the stepthrough latency time (s) was significantly decreased compared to the sham group $(p<0.001)$. However, administration of PRE at doses of 100 and $300 \mathrm{mg} / \mathrm{kg}$ significantly improved the step-through latency time (s). These effects were similar to those observed for St. John's wort $(p<0.05)$.

\section{Effect of PRE on TST and FST}

Repeated CORT injections had a significant effect on mice behavior in the tail suspension test (TST) and forced swim test (FST) (Badr et al., 2020). The CORT-treated control group exhibited depression-like behavior characterized by significantly increased immobility time in TST and FST. An increase in immobility time is interpreted as depression-like behavior in animal models of depression (Renard et al., 2003). As expected, the group that was administered St. John's wort extract at a dose of $300 \mathrm{mg} / \mathrm{kg}$ showed a significant decrease in immobility time compared to the control group, which was similar to previously published preclinical studies (Crupi et al., 2013). Similar efficacy was exhibited by the administration of PRE at dose of $300 \mathrm{mg} / \mathrm{kg}$ in the TST and FST (Fig. 6A and 6C). These results indicate that administration of PRE had antidepressant-like effects in a CORT-induced depression model.

\section{Effect of PRE on GR activity}

Prolonged or excessive exposure to CORT leads to neuronal damage, particularly in the hippocampus, which is enriched with glucocorticoid receptor (GR) (B. J. Liu et al., 2011). After the FST, the hippocampal tissue from each mouse were extracted from the whole brain for western blot analysis. Depression has been correlated with alternations in GR signaling (Jovicic et al., 2015). GR can be phosphorylated at serine 211 (Ser211) and enhanced Ser211 had a stronger correlation with depression (Bei et al., 2009). Indeed, chronic glucocorticoids stress, which stimulates GR activation, causes downregulation of expression of brain-derived neurotrophic factor (BDNF) (Numakawa et al., 2017). As shown in Fig. 7, the hippocampal BDNF levels were significantly decreased, whereas total GR (tGR) and GR phosphorylation at Ser211, were significantly increased in the CORT-treated control group. However, PRE at a dose of 300 $\mathrm{mg} / \mathrm{kg}$ significantly increased BDNF levels and decreased tGR and Ser211 levels within the hippocampus. These results indicate that administration of PRE improved the overexpression of the GR receptor by CORT-induced neurotoxicity and increased the activity of BDNF, thereby alleviating depression and improving cognitive function.

\section{Effect of PRE on cell viability}

We examined the potential neuroprotective effects of PRE on CORT-induced toxicity, a model that mimics glucocorticoid hypersecretion in neuronal cell (SH-SY5Y) which is known to facilitate neurotoxicity (Lim et al., 2018b). Application of CORT (200 区) to SH-SY5Y cells resulted in a significant reduction in cell viability by approximately $50 \%$ compared with normal cells (Con) (Fig. 8A), and this reduction was significantly prevented by co-treatment with PRE (Fig. 8B). These result indicated that PRE can protect against CORT-induced neurotoxicity in neuronal cell. 


\section{Discussion}

In the present study, we determined that PRE administration was sufficient to induce antidepressant-like effects in a well-established CORT-induced mouse model of depression-like (Zhao et al., 2008). We found that PRE administration facilitated antidepressant-like behavior and significantly reduced immobility time in the TST or FST, and increased sucrose consumption without affecting locomotor activity. Moreover, PRE attenuated the CORT-induced abnormal activation of GRs and improved cognitive function by increasing BDNF levels. These neuroprotective effects were also confirmed in human neuroblastoma SHSY5Y cells.

Although the complex pathogenesis of depression remains poorly understood, HPA axis dysfunction is a well-known risk factor for depression, including cognitive impairment (Karstens et al., 2019). The fact that patients with depression exhibit cortisol hypersecretion due to disturbance of the glucocorticoid negative feedback system (Mizoguchi et al., 2003) suggests an association between HPA axis dysfunction and depression (Jia et al., 2019). It has also been reported that exposure to high levels of CORT cause damage to the brain, particularly the hippocampus, one of the brain regions where GR are highly concentrated (Mason and Pariante, 2006), leading to symptoms such as cognitive decline or depression. Patients with depression have been reported to have smaller hippocampal volume compared to healthy controls (Colle et al., 2016). Similarly, in animal studies, chronic stress or repeated injections of high-dose CORT induce HPA axis dysfunction, resulting in depression-like behavior, which is modulated by antidepressant treatment (lijima et al., 2010; Naert et al., 2011). Immobility in behavioral tasks related to animal models of depression has been coined as behavioral despair or learned helplessness (Castagne et al., 2011). Increased immobility in the TST or FST is well known to parallel human depression-related symptomology, and this state of immobility is significantly improved when treated with antidepressants (Y. Zhang et al., 2017). Sucrose consumption in animals has been used as an indicator of anhedonia-like behavior. Anhedonia is a core symptom of major depression in humans (M. Y. Liu et al., 2018). We determined that immobility time was significantly increased in the TST and FST as well as decreased sucrose consumption in mice receiving $40 \mathrm{mg} / \mathrm{kg}$ of CORT daily, and this study showed a similar trend to that of a previous study (Lim et al., 2019b). As expected, we found that the PRE-treated group had significantly reduced immobility time in the TST and FST without any changes in locomotor activity, especially at the $300 \mathrm{mg} / \mathrm{kg}$ dose; this antidepressant-like effect was similar to that of the group receiving St. John's wort extract as a positive control. Moreover, PRE significantly increased the preference for sucrose consumption. These results suggest that PRE has antidepressant-like effects in CORT-induced depression animal model.

GR is a phosphoprotein that becomes hyper-phosphorylated upon binding to glucocorticoids (Quax et al., 2013). Excessive GR activation by high concentrations of CORT impairs hippocampal neurogenesis, suggesting that normalization of GR function is the critical to the antidepressant effect (Pariante, 2004). It has been reported that the expression of hippocampal GR is significantly upregulated by exposure to chronic mild stress-induced depression in animal models (Wei et al., 2016). GR activation is critically dependent on the phosphorylation status of specific serine residues such as Ser211 (Anacker et al., 
2011). It has been reported that phosphorylation at Ser211 facilitates nuclear translocation and increases GR transcriptional activity (K. Zhang et al., 2019). Our results also showed that total GR protein levels were increased by chronic CORT exposure, suggesting that prolonged exposure to CORT resulted in the upregulation of GR expression and increased GR phosphorylation at Ser211, whereas PRE normalized the CORT-induced increase in GR protein and GR phosphorylation at Ser211. BDNF, a member of the neurotrophin family, is highly expressed in the hippocampus and is mainly involved in cognitive function and mood changes (Teixeira et al., 2010). Clinical studies have demonstrated that BDNF is an important factor in the pathogenesis of depression (Fernandes et al., 2011). It has been reported that low serum BDNF levels in patients with depression, were significantly increased by antidepressant treatment (Shimizu et al., 2003). We determined that the level of hippocampal BDNF was significantly decreased in the CORT-treated control group, which was correlated with cognitive decline in the passive avoidance test (PAT), following CORT injection in mice. As expected, we found that PRE prevented the CORT-induced reduction in BDNF levels in the hippocampus, and PRE treatment promoted cognitive improvement evidenced by increasing step through latency time in the PAT.

A limitation of this study is that our in vivo findings present a portion of the animal's behavior results, and the dose does not reveal the bioavailability of the active compounds of PRE. Thus, the efficacy of the active PRE compounds needs to be tested in vivo. Moreover, further studies are necessary to determine whether active compounds from PRE act on the central nervous system through blood-brain barrier penetration.

Taken together, our results indicate that PRE upregulates BDNF levels against CORT-induced depressionlike phenotypes by regulating the HPA axis through normalizing GR function via inhibition of Ser211mediated GR phosphorylation.

\section{Conclusion}

To our knowledge, this is the first study evaluating the antidepressant-like effects of $P$. resupinata extract on CORT-induced depressive-like behaviors in mice. We found that $P$. resupinata extract prevented CORTinduced depression-like behavior and that this effect may be mediated by the regulation of GR and BDNF levels, which are involved in the inhibition of neuronal loss.

\section{Declarations}

Author Contributions D.W.L. and C.L. designed the study. D.W.L. and D.H. performed the HPLC analysis and cell and animal experiments. D.W.L. and C.L. wrote the manuscript. D.H. and C.L. contributed expert opinions to the manuscript correction. All authors have read and agreed to the published version of the manuscript.

Funding: This research was funded by the Main Research Program of the Korea Food Research Institute, funded by the Ministry of Science and ICT (grant number E0210201-01). 
Data Availability The data presented in this study are available on request from the corresponding authors.

\section{Conflict of interest}

There was no conflict of interest of all authors.

\section{Ethical approval}

The study was conducted according to the guidelines of the Korea Food Research Institute, and approved by the Institutional Animal Care and Use Committee of the Korea Food Research Institute (protocol code KFRI-M-19016).

\section{References}

1. Ali AM, Alkhamees AA, Hori H, Kim Y, Kunugi H (2021) The Depression Anxiety Stress Scale 21: Development and Validation of the Depression Anxiety Stress Scale 8-Item in Psychiatric Patients and the General Public for Easier Mental Health Measurement in a Post COVID-19 World.International Journal of Environmental Research and Public Health18

2. Anacker C, Zunszain PA, Cattaneo A, Carvalho LA, Garabedian MJ, Thuret S et al (2011) Antidepressants increase human hippocampal neurogenesis by activating the glucocorticoid receptor. Mol Psychiatry 16:738-750

3. Badr AM, Attia HA, Al-Rasheed N (2020) Oleuropein Reverses Repeated Corticosterone-Induced Depressive-Like Behavior in mice: Evidence of Modulating Effect on Biogenic Amines. Sci Rep 10:3336

4. Bei E, Salpeas V, Pappa D, Anagnostara C, Alevizos V, Moutsatsou P (2009) Phosphorylation status of glucocorticoid receptor, heat shock protein 70, cytochrome $\mathrm{c}$ and Bax in lymphocytes of euthymic, depressed and manic bipolar patients. Psychoneuroendocrinology 34:1162-1175

5. Castagne V, Moser P, Roux S, Porsolt RD (2011) Rodent models of depression: forced swim and tail suspension behavioral despair tests in rats and mice.Curr Protoc Neurosci Chap. 8, Unit $810 \mathrm{~A}$.

6. Colle R, Cury C, Chupin M, Deflesselle E, Hardy P, Nasser G et al (2016) Hippocampal volume predicts antidepressant efficacy in depressed patients without incomplete hippocampal inversion. Neuroimage-Clinical 12:949-955

7. Crupi R, Abusamra YAK, Spina E, Calapai G (2013) Preclinical Data Supporting/Refuting the Use of Hypericum perforatum in the Treatment of Depression. Cns \& Neurological Disorders-Drug Targets 12:474-486

8. Cui QL, Pan YN, Zhang W, Zhang YA, Ren SM, Wang DM et al (2018) Metabolites of Dietary Acteoside: Profiles, Isolation, Identification, and Hepatoprotective Capacities. J Agric Food Chem $66: 2660-2668$ 
9. Fernandes BS, Gama CS, Cereser KM, Yatham LN, Fries GR, Colpo G et al (2011) Brain-derived neurotrophic factor as a state-marker of mood episodes in bipolar disorders: A systematic review and meta-regression analysis. J Psychiatr Res 45:995-1004

10. lijima M, Ito A, Kurosu S, Chaki S (2010) Pharmacological characterization of repeated corticosterone injection-induced depression model in rats. Brain Res 1359:75-80

11. Jia Y, Liu L, Sheng C, Cheng Z, Cui L, Li M et al (2019) Increased Serum Levels of Cortisol and Inflammatory Cytokines in People With Depression. J Nerv Ment Dis 207:271-276

12. Jovicic M, Maric NP, Soldatovic I, Lukic I, Andric S, Mihaljevic M et al (2015) The role of glucocorticoid receptor phosphorylation in the model of negative affective states. World J Biol Psychiatry 16:301-311

13. Kalueff AV, Murphy DL (2007) The importance of cognitive phenotypes in experimental modeling of animal anxiety and depression. Neural Plast 2007, 52087

14. Karstens AJ, Korzun I, Avery ET, Kassel MT, Keelan R, Kales H et al (2019) Examining HPA-axis functioning as a mediator of the relationship between depression and cognition across the adult lifespan. Neuropsychol Dev Cogn B Aging Neuropsychol Cogn 26:507-520

15. Lee HD, Kim JH, Pang QQ, Jung PM, Cho EJ, Lee S (2020) Antioxidant Activity and Acteoside Analysis of Abeliophyllum distichum. Antioxidants 9

16. Lee YM, Jung HY, Bae JH, Lee JY, Jang HH, Park DS et al (2011) Screening of Korean edible wild plants for estrogenic activity using MCF-7 cell proliferation assay. Food Sci Biotechnol 20:829-833

17. Lim DW, Han T, Jung J, Song Y, Um MY, Yoon M et al (2018a) Chlorogenic Acid from Hawthorn Berry (Crataegus pinnatifida Fruit) Prevents Stress Hormone-Induced Depressive Behavior, through Monoamine Oxidase B-Reactive Oxygen Species Signaling in Hippocampal Astrocytes of Mice, vol 62. Molecular Nutrition \& Food Research

18. Lim DW, Han T, Um MY, Yoon M, Kim TE, Kim YT et al (2019a) Administration of Asian Herb Bennet (Geum japonicum) Extract Reverses Depressive-Like Behaviors in Mouse Model of Depression Induced by Corticosterone. Nutrients 11 .

19. Lim DW, Han T, Um MY, Yoon M, Kim TE, Kim YT et al (2019b) Administration of Asian Herb Bennet (Geum japonicum) Extract Reverses Depressive -Like Behaviors in Mouse Model of Depression Induced by Corticosterone. Nutrients 11 .

20. Lim DW, Um MY, Han T, Lee J, Kim YT, Cho S et al (2018b) Standardized Citrus unshiu peel extract ameliorates dexamethasone-induced neurotoxicity and depressive-like behaviors in mice. Metab Brain Dis 33:1877-1886

21. Liu BJ, Zhang HY, Xu CQ, Yang GA, Tao JA, Huang JH et al (2011) Neuroprotective effects of icariin on corticosterone-induced apoptosis in primary cultured rat hippocampal neurons. Brain Res 1375:59-67

22. Liu MY, Yin CY, Zhu LJ, Zhu XH, Xu C, Luo CX et al (2018) Sucrose preference test for measurement of stress-induced anhedonia in mice. Nat Protoc 13:1686-1698 
23. Liu XQ, Yan Y, Li F, Zhang DF (2016) Fruit and vegetable consumption and the risk of depression: A meta-analysis. Nutrition 32:296-302

24. Mason BL, Pariante CM (2006) The effects of antidepressants on the hypothalamic-pituitary-adrenal axis. Drug News Perspect 19:603-608

25. Mizoguchi K, Ishige A, Aburada M, Tabira T (2003) Chronic stress attenuates glucocorticoid negative feedback: involvement of the prefrontal cortex and hippocampus. Neuroscience 119:887-897

26. Naert G, Ixart G, Maurice T, Tapia-Arancibia L, Givalois L (2011) Brain-derived neurotrophic factor and hypothalamic-pituitary-adrenal axis adaptation processes in a depressive-like state induced by chronic restraint stress. Mol Cell Neurosci 46:55-66

27. Numakawa T, Odaka H, Adachi N (2017) Actions of Brain-Derived Neurotrophic Factor and Glucocorticoid Stress in Neurogenesis.International Journal of Molecular Sciences18

28. Pariante CM (2004) Glucocorticoid receptor function in vitro in patients with major depression. Stress-the Int J Biology Stress 7:209-219

29. Pirotta M, Willis K, Carter M, Forsdike K, Newton D, Gunn J (2014) 'Less like a drug than a drug': The use of St John's wort among people who self-identify as having depression and/or anxiety symptoms. Complement Ther Med 22:870-876

30. Qiao ZG, Tang JX, Wu W, Tang J, Liu M (2019) Acteoside inhibits inflammatory response via JAK/STAT signaling pathway in osteoarthritic rats.Bmc Complementary and Alternative Medicine19

31. Quax RA, Manenschijn L, Koper JW, Hazes JM, Lamberts SWJ, van Rossum EFC et al (2013) Glucocorticoid sensitivity in health and disease. Nat Reviews Endocrinol 9:670-686

32. Racagni G, Popoli M (2010) The pharmacological properties of antidepressants. Int Clin Psychopharmacol 25:117-131

33. Renard CE, Dailly E, David DJ, Hascoet M, Bourin M (2003) Monoamine metabolism changes following the mouse forced swimming test but not the tail suspension test. Fundam Clin Pharmacol 17:449-455

34. Shimizu E, Hashimoto K, Okamura N, Koike K, Komatsu N, Kumakiri C et al (2003) Alterations of serum levels of brain-derived neurotrophic factor (BDNF) in depressed patients with or without antidepressants. Biol Psychiatry 54:70-75

35. Teixeira AL, Barbosa IG, Diniz BS, Kummer A (2010) Circulating levels of brain-derived neurotrophic factor: correlation with mood, cognition and motor function. Biomark Med 4:871-887

36. Wang HQ, Xu YX, Yan J, Zhao XY, Sun XB, Zhang YP et al (2009) Acteoside protects human neuroblastoma SH-SY5Y cells against beta-amyloid-induced cell injury. Brain Res 1283:139-147

37. Wang P, Kang J, Zheng R, Yang Z, Lu J, Gao J et al (1996) Scavenging effects of phenylpropanoid glycosides from Pedicularis on superoxide anion and hydroxyl radical by the spin trapping method(95)02255-4. Biochem Pharmacol 51:687-691

38. Wei K, Xu YZ, Zhao ZX, Wu X, Du YJ, Sun J et al (2016) Icariin alters the expression of glucocorticoid receptor, FKBP5 and SGK1 in rat brains following exposure to chronic mild stress. Int J Mol Med 
38:337-344

39. Zhang K, He MY, Wang F, Zhang HT, Li YT, Yang JY et al (2019) Revealing Antidepressant Mechanisms of Baicalin in Hypothalamus Through Systems Approaches in Corticosterone-Induced Depressed Mice.Frontiers in Neuroscience13

40. Zhang $Y$, Wang $Y T$, Lei $H$, Wang $L$, Xue L, Wang $X$ et al (2017) Optimized animal model to mimic the reality of stress-induced depression in the clinic.Bmc Psychiatry 17

41. Zhao Y, Ma R, Shen J, Su H, Xing DM, Du LJ (2008) A mouse model of depression induced by repeated corticosterone injections. Eur J Pharmacol 581:113-120

\section{Figures}
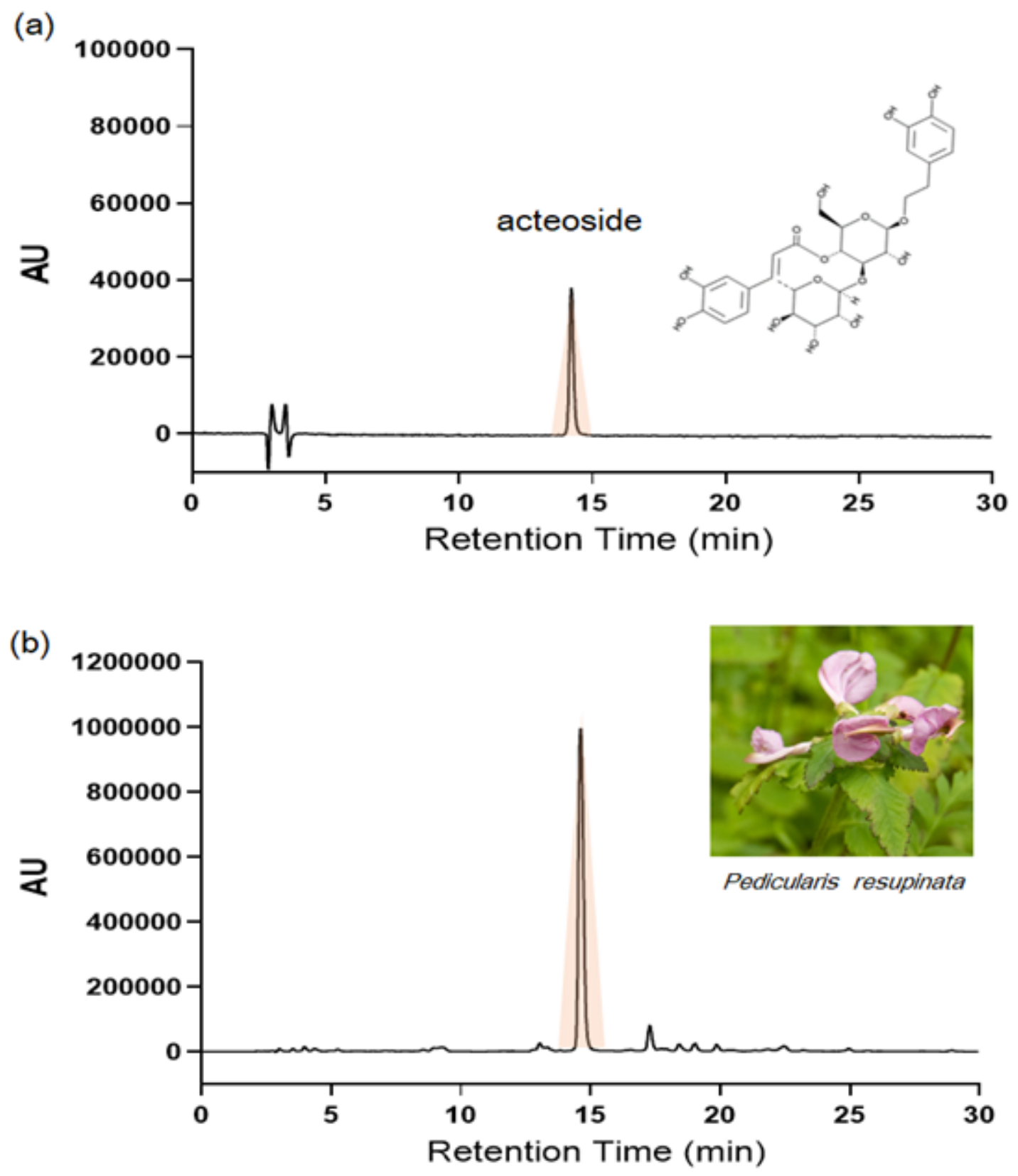


\section{Figure 1}

HPLC chromatogram of (a) acteoside as a standard compound and (b) Pedicularis resu-pinata extract. The concentration of acteoside was $0.78 \pm 1.15 \mathrm{mg} / \mathrm{g}$ PRE.

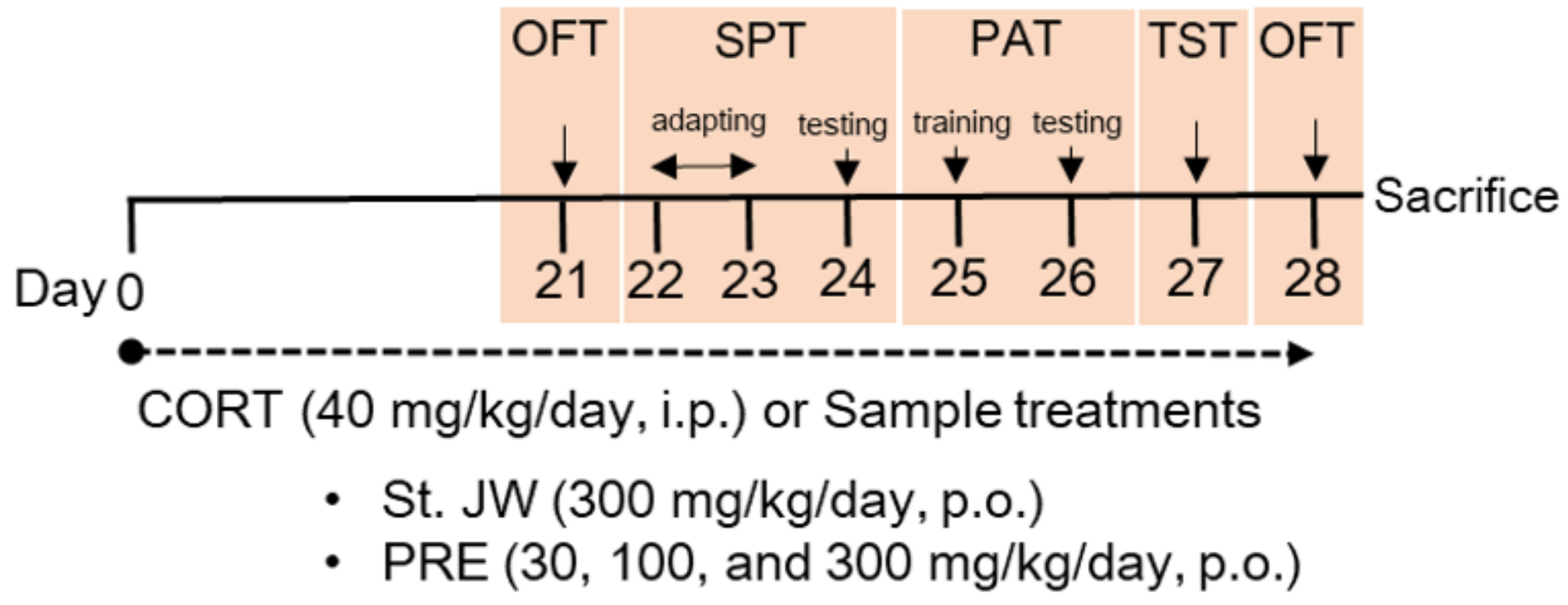

Figure 2

Experimental design for animal model of CORT-induced depression-like behavior. CORT, corticosterone; OFT, open-field test; SPT, Sucrose Preference Test; PAT, passive avoidance test; TST, tail suspension test; FST, forced swim test; St. JW, Saint John's wort extract; PRE, P. resupinata extract. 


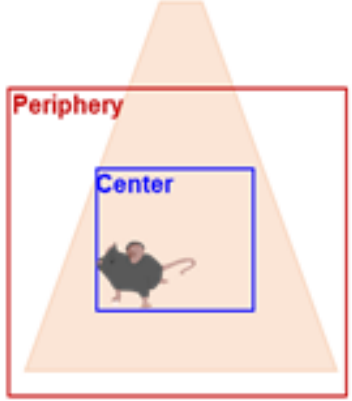

(a)

Sham_vehicle

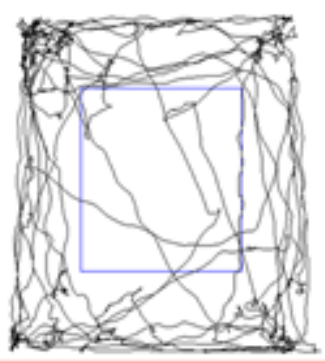

CORT_Con_vehicle

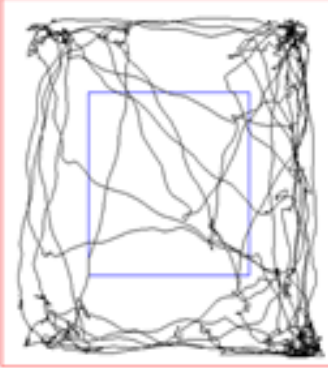

\section{CORT_PRE $300 \mathrm{mg} / \mathrm{kg}$}

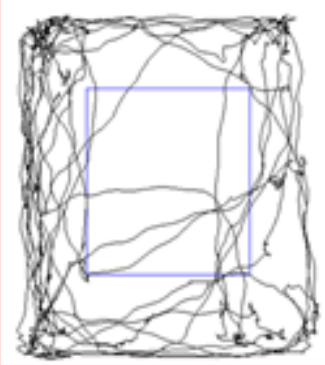

(b)

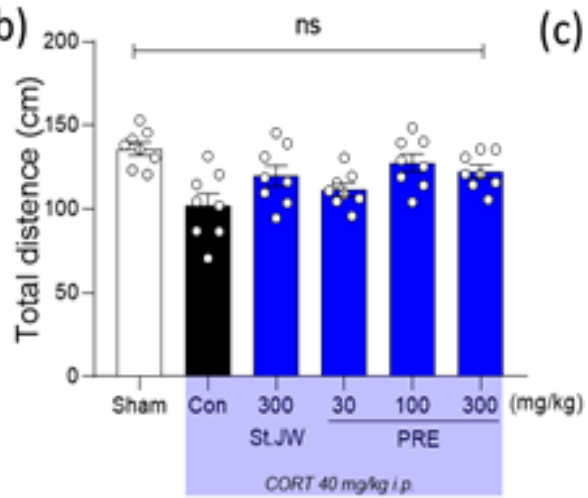

(c)

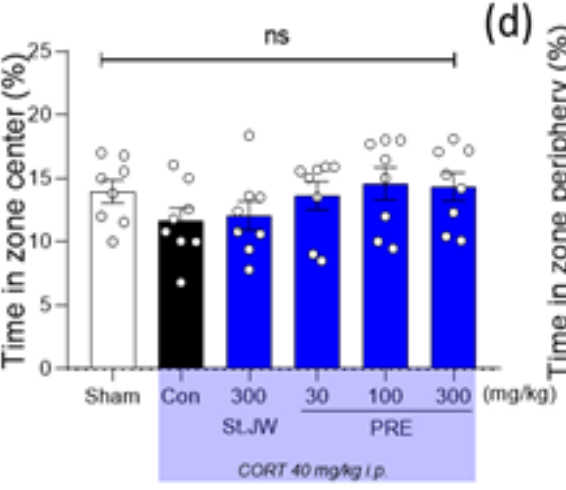

(d)

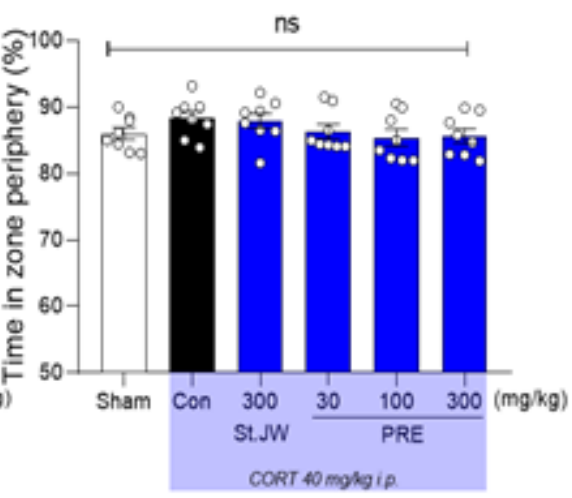

Figure 3

Effect of PRE on open filed test in CORT-induced depressive mice. A representative trace of locomotor activity across $5 \mathrm{~min}(\mathrm{~A})$ in the OFT. There was no significant difference in total distance moved (B), time spent in the center (C), or periphery (D) of the OFT, between the treated groups. Results are presented as mean \pm SEM ( $n=8$, per group). Differences among experimental groups were determined by analysis of variance (ANOVA) test. Con, control; CORT, corticosterone; St.JW, Saint John's wort extract; PRE, P. resupinata extract. 


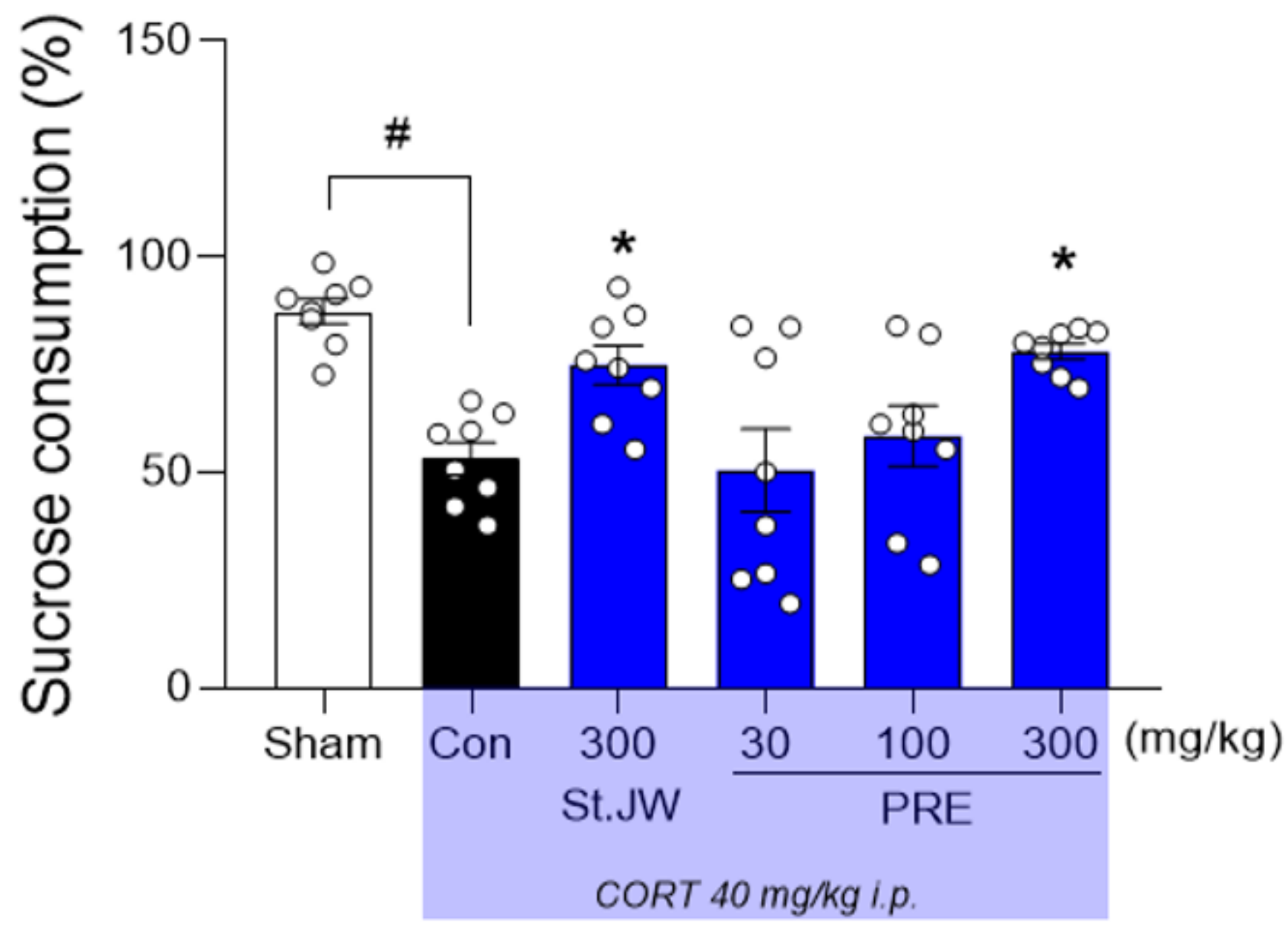

Figure 4

Effect of PRE on sucrose consumption in CORT-induced depressive mice. CORT injections significantly decreased sucrose consumption, while treatment with PRE at doses of $300 \mathrm{mg} / \mathrm{kg}$ significantly increased it. Results are presented as mean \pm SEM ( $n=8$, per group). $\# p<0.05$ versus the sham group; ${ }^{*} p<0.05$ versus the CORT-injected Con group. Con, control; CORT, corticosterone; St.JW, Saint John's wort extract; $\mathrm{PRE}, P$. resupinata extract. 


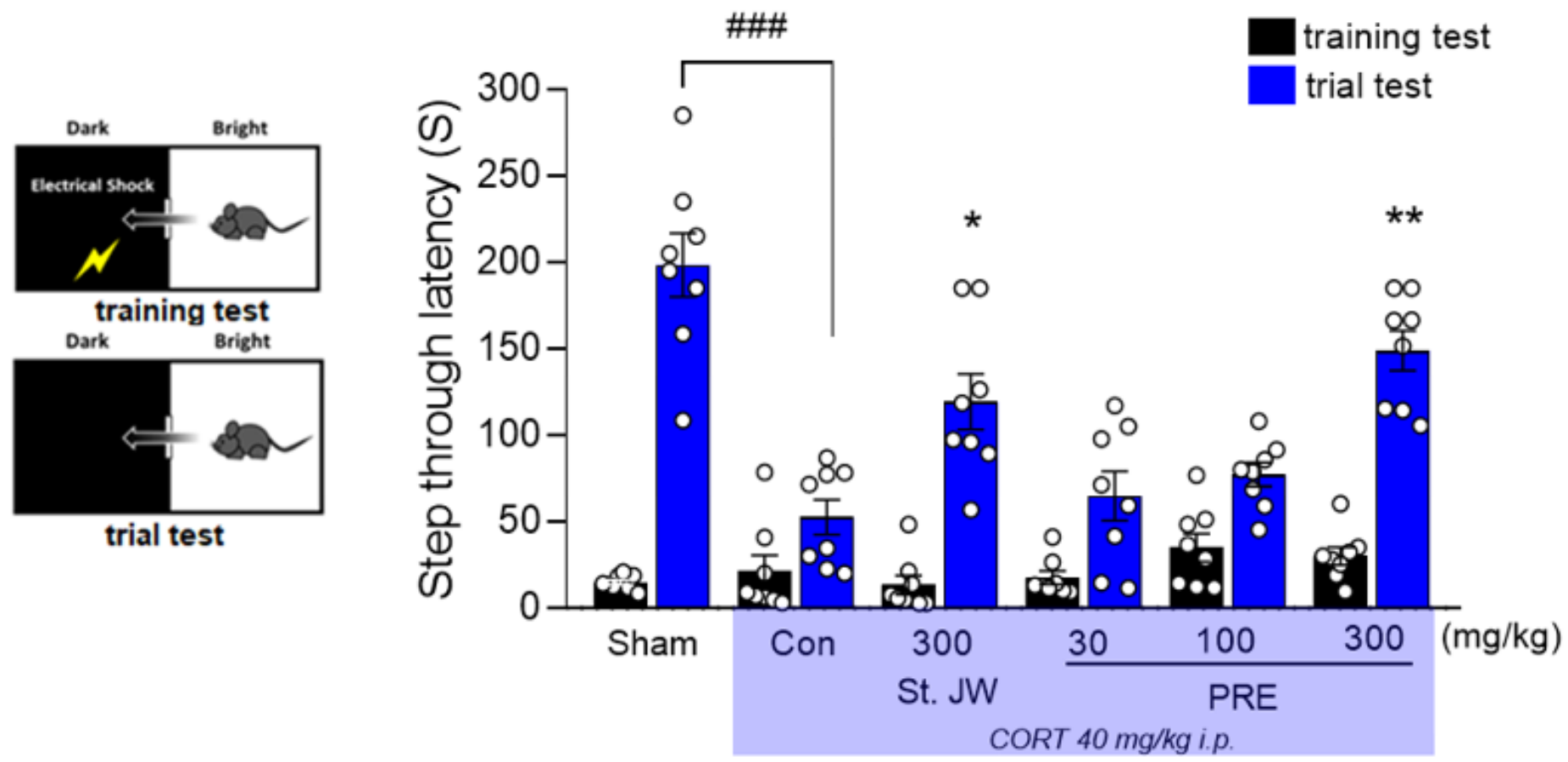

Figure 5

Effect of PRE on passive avoidance test in CORT-induced depressive mice. CORT injections significantly decreased step-through latency time (s), while administration of PRE at doses of $300 \mathrm{mg} / \mathrm{kg}$ was significant increased latency time. Results are presented as mean $\pm \operatorname{SEM}(n=8$, per group). \#\#\# $p<0.001$ versus the sham group; * $p<0.05$ and ${ }^{\star \star} p<0.01$ versus the CORT-injected Con group. Con, control; CORT, corticosterone; St.JW, Saint John's wort extract; PRE, P. resupinata extract. 

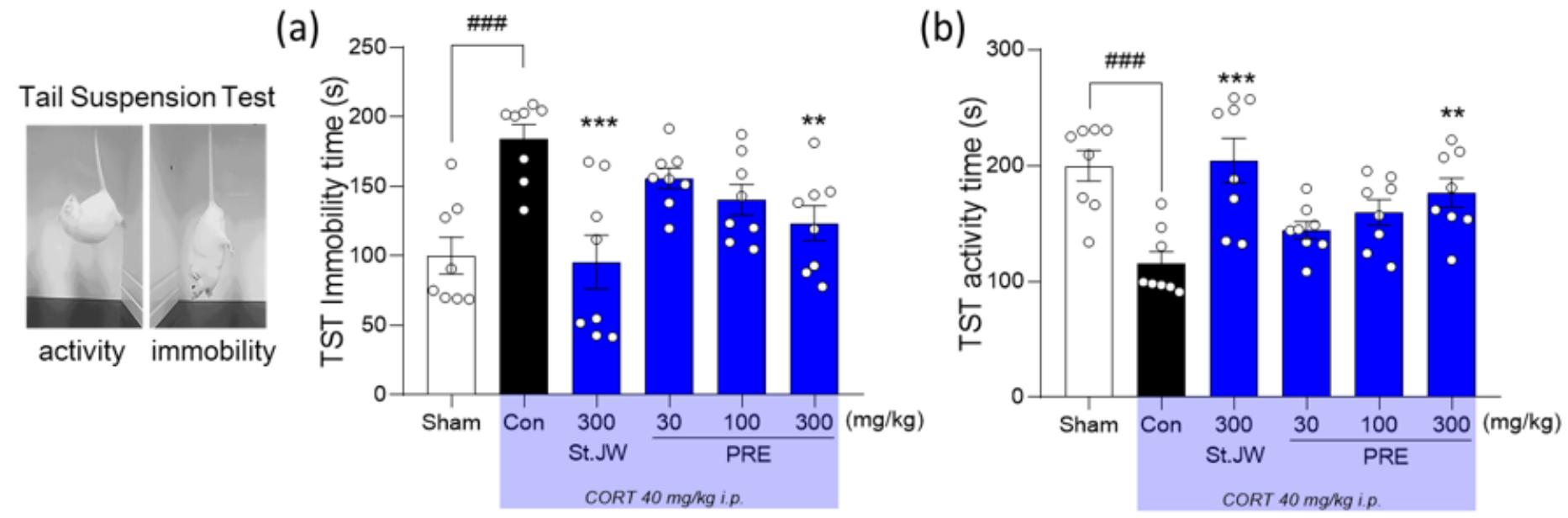

(c)

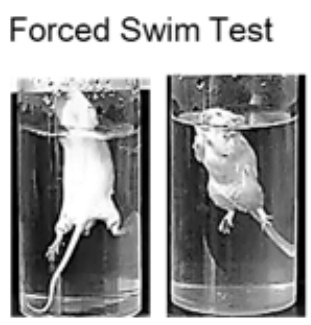

swimming immobility

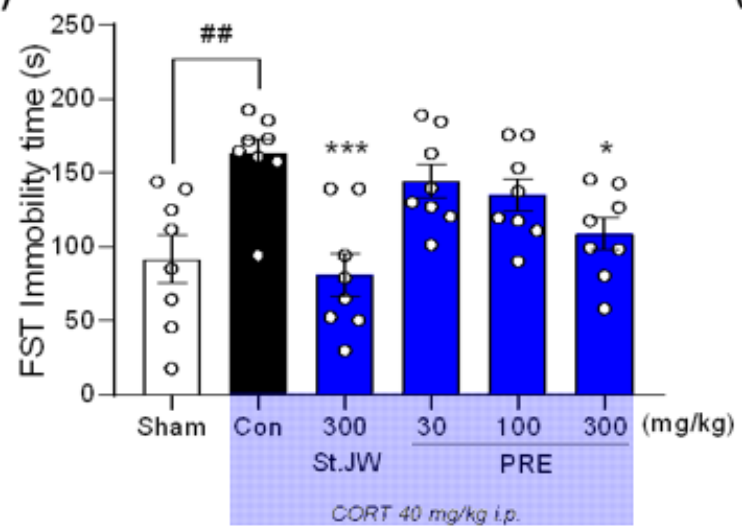

(d)

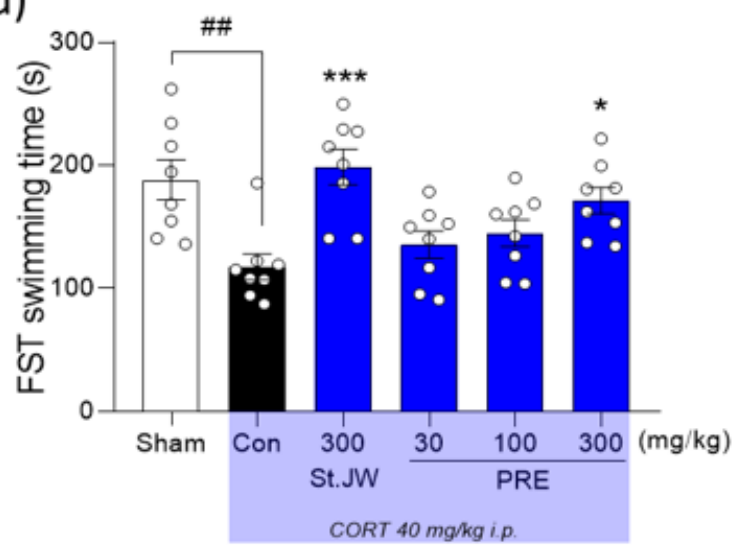

Figure 6

Effect of PRE on tail suspension test and forced swim test in CORT-induced depressive mice. CORTinjected mice had significantly increased immobility and decreased activity, including of swimming, while PRE at dose of $300 \mathrm{mg} / \mathrm{kg}$ treated-mice showed significant improvements, with a decreased immobility (A and C) times (s) and increased activity (B) and swimming (D) times (s). Results are presented as mean \pm SEM ( $n=8$, per group). \#\# $p<0.01$ and \#\#\# $p<0.001$ versus the sham group; ${ }^{*} p<0.05$, $* \star p<0.01$, and $\star \star p<0.001$ versus the CORT-injected Con group. Con, control; CORT, corticosterone; St.JW, Saint John's wort extract; PRE, P. resupinata extract. 

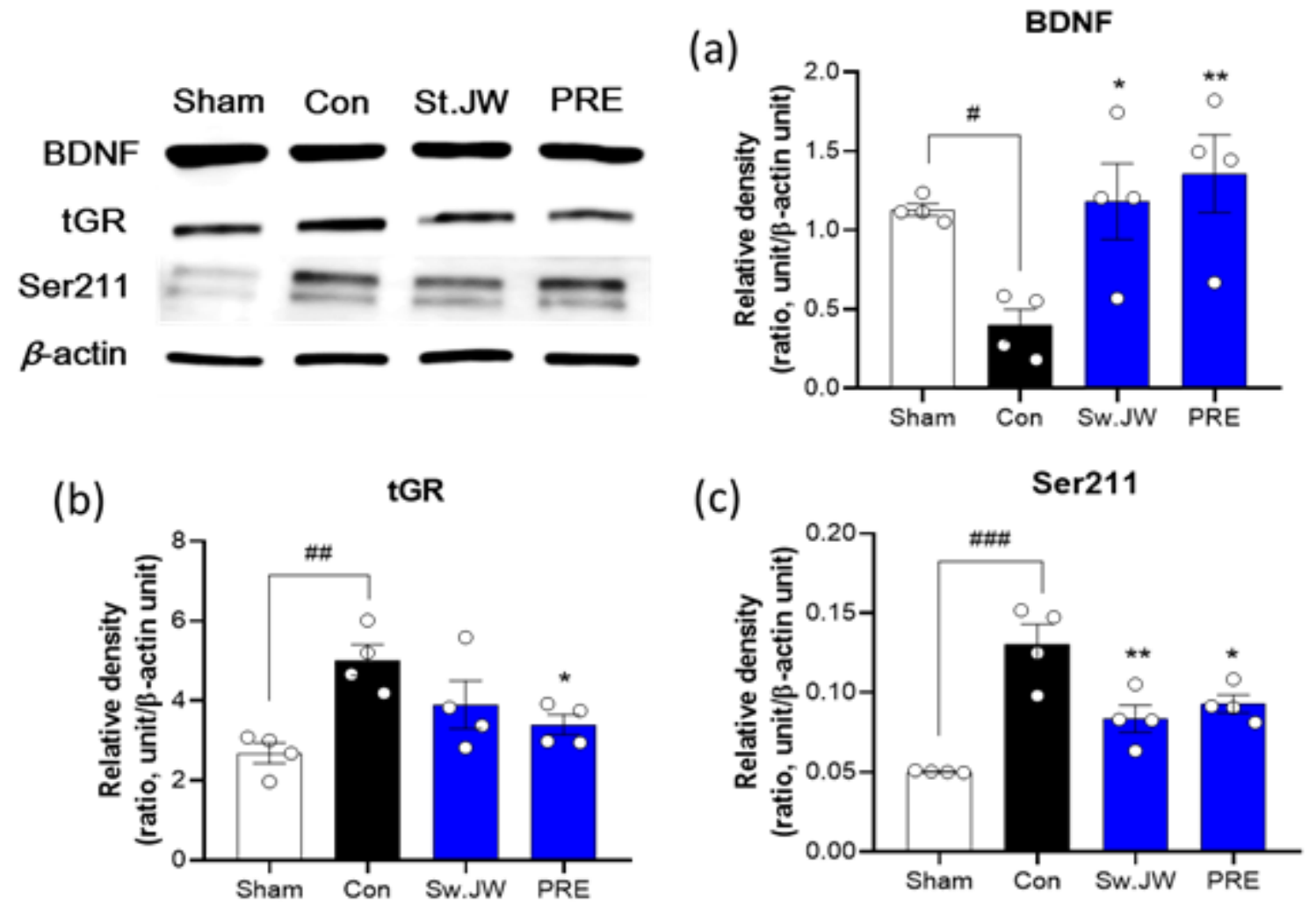

Figure 7

Effect of PRE on glucocorticoid receptor activity in CORT-induced depressive mice. Hippocampal western blot analysis revealed that CORT injections significantly decreased BDNF (A) expression, and increased tGR (B) and Ser211 (C) expression in the hippocampus, while treatment with PRE at $300 \mathrm{mg} / \mathrm{kg}$ significantly attenuated the effect. Results are presented as mean $\pm \operatorname{SEM}(\mathrm{n}=4$, per group). \# $p<0.05$, \#\#

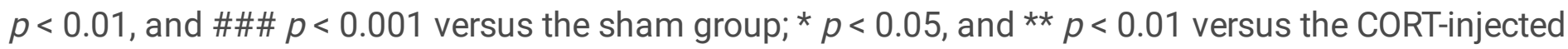
Con group. Con, control; CORT, corticosterone; St.JW, Saint John's wort extract; PRE, P. resupinata extract. 
(a)

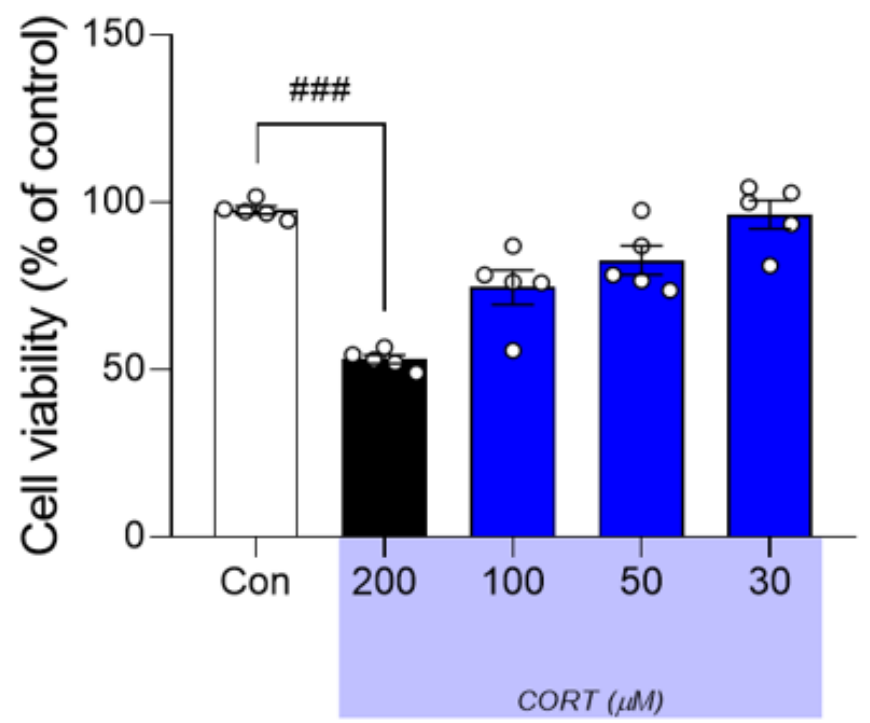

(b)

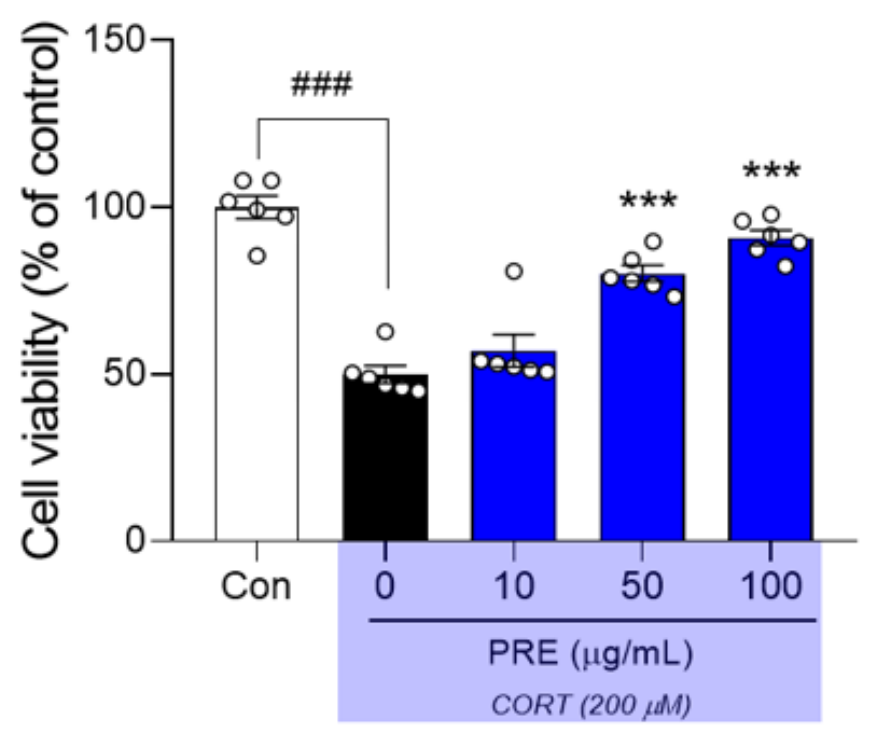

\section{Figure 8}

Effect of PRE on CORT-induced neurotoxicity in SH-SY5Y cells. Reduction of cell viability was prevented by co-treatment with PRE in SH-SY5Y cells. Results are presented as mean $\pm \mathrm{SEM}$ ( $\mathrm{n}=6$, per group); \#\#\# $p$ $<0.001$ versus Normal group; $* \star \star ~ p<0.001$ versus the CORT-injected Con group; Con, control; CORT, corticosterone; PRE, P. resupinata extract. 